\title{
Mapping speed for an array of corrugated horns
}

\author{
Stephen Padin \\ California Institute of Technology MC367-17, Pasadena, California 91125, USA \\ (spadin@ caltech.edu)
}

Received 19 June 2009; revised 3 December 2009; accepted 21 December 2009; posted 22 December 2009 (Doc. ID 113071); published 15 January 2010

\begin{abstract}
I address the choice of horn diameter for millimeter-wave array receivers with corrugated horns. For maximum point-source mapping speed, in both total power and polarization with typical receiver noise contributions and a close-packed horn array that fills the field of view, the optimum horn diameter is $1.6-1.7 F \lambda$, where $F$ is the focal ratio. A $\pm 25 \%$ change in horn diameter gives $<10 \%$ degradation in mapping speed. Correlated noise from the cold stop, atmosphere, and cosmic microwave background has little effect on the mapping speed and optimum horn diameter. (C) 2010 Optical Society of America

OCIS codes: $\quad 040.1240,040.2235,350.1260,350.4010$.
\end{abstract}

\section{Introduction}

Corrugated horns are an attractive option for coupling receivers to a telescope because they offer small polarization errors and good control of the beam shape [1]. These features are important for cosmic microwave background (CMB) instruments, especially polarimeters. Measurements of CMB polarization anisotropy provide an important window on the early universe $[2,3]$. The gravitational wave background from inflation and gravitational lensing by large-scale structures should both leave unique signatures in the polarization anisotropy [4, $\underline{5}$ ]. These signals are at most a few $\times 100 \mathrm{nK}$, so observations require high sensitivity and small systematic errors. CMB polarimeters, therefore, have large arrays of receivers coupled to telescopes that are optimized for low scattering [ㅁ-으. Existing corrugated horn arrays are relatively sparse, so the horns are designed for maximum aperture efficiency. New receivers will have arrays that completely fill the field of view (FOV) of the telescope. In this case, sensitivity can be improved by making the horns smaller in order to fit more horns in the FOV.

To design an array receiver with corrugated horns, we need to know how the horn aperture diameter affects the sensitivity or mapping speed. This calculation is not trivial because the photon bunching

0003-6935/10/030479-05\$15.00/0

(C) 2010 Optical Society of America noise [9-12] is correlated between horns. Details of the mapping speed calculation are presented here. The material is organized as follows: Section 2 gives the mapping speed for single-pixel and array receivers in terms of general efficiency and noise parameters; Section 3 contains results for millimeterwave, total-powe $\bar{r}$ receivers and polarimeters with corrugated horns; and Section $\underline{4}$ is a summary.

\section{Mapping Speed}

In this section, I estimate the point-source mapping speed for single-pixel and array receivers. The pointsource mapping speed is an important measure because $\mathrm{CMB}$ observations usually require information at the resolution limit of the telescope in order to remove foregrounds.

Consider first a single receiver that responds to one spatial mode and one or two polarizations, e.g., a horn and detector behind a bandpass filter. The horn looks at a cold stop (a cold box completely surrounding the pupil), which defines the illumination on the telescope aperture. The telescope optics transform the field at the stop to a beam on the sky. The signal at the horn input due to a point source is

$$
S \propto \eta_{a}
$$

where $\eta_{a}$ is the aperture efficiency (e.g., the overlap of the horn and Airy pattern fields). The signal also depends on the transmission of the atmosphere and the optical efficiency of the telescope. I will assume these 
are the same for all receiver configurations, so they do not affect the choice of horn diameter. The noise equivalent power at the horn input is given by [13]

$$
\begin{aligned}
\mathrm{NEP}_{1}^{2}= & \eta_{s}\left(\mathrm{NEP}_{\mathrm{cmb}}^{2}+\mathrm{NEP}_{\mathrm{atm}}^{2}\right) \\
& +\left(1-\eta_{s}\right) \mathrm{NEP}_{\text {stop }}^{2}+\mathrm{NEP}_{\text {det }}^{2}
\end{aligned}
$$

where $\eta_{s}$ is the spillover efficiency, i.e., the fraction of the beam from the horn that passes through the stop. $\mathrm{NEP}_{\mathrm{cmb}}, \mathrm{NEP}_{\mathrm{atm}}$, and $\mathrm{NEP}_{\text {stop }}$ are photon noise contributions from the CMB, atmosphere (and telescope) and the stop. These terms include the transmission efficiency for the instrument and the detector quantum efficiency. $\mathrm{NEP}_{\text {det }}$ represents noise from the detector and readout.

The photon noise for an unpolarized source is given by $[\underline{11}, \underline{12}, \underline{14}-\underline{16}]$

$$
\mathrm{NEP}^{2}=2 \int Q h \nu \mathrm{d} \nu+\int Q^{2} \mathrm{~d} \nu
$$

e.g., in $\mathrm{W}^{2} \mathrm{~Hz}^{-1}$, where $Q$ is the source spectral density, $h$ is Planck's constant, and $\nu$ is the frequency. The two terms in Eq. (3) represent the Poisson (shot) noise, $\mathrm{NEP}_{P}^{2}=2 \int Q \bar{h} \nu \mathrm{d} \nu$, and the Bose (photon bunching) noise, $\mathrm{NEP}_{B}^{2}=\int Q^{2} \mathrm{~d} \nu$. For a thermal source

$$
Q=q \epsilon \frac{h \nu}{\exp (h \nu / k T)-1},
$$

where $q$ is the number of polarizations detected, $\epsilon$ and $T$ are the emissivity and temperature of the source, and $k$ is Boltzmann's constant. In the Rayleigh-Jeans limit, Eq. (3) gives, as expected, $\mathrm{NEP}=q € k T B^{1 / 2}$, where $B$ is the bandwidth. Table 1 shows NEP contributions for a typical millimeter-wave receiver. All the Bose noise contributions are significant, and they can be correlated between horns in an array.

The point-source mapping speed for a single horn is [13]

$$
M_{1} \propto \frac{S^{2}}{\mathrm{NEP}_{1}^{2}}
$$

If the noise is uncorrelated between horns, the mapping speed for an array is

$$
M_{\text {array }}=n M_{1},
$$

where $n$ is the number of horns in the array. The effect of correlations in the noise can be included by summing $\mathrm{NEP}^{2}$ over the entire array and calculating an effective $\mathrm{NEP}^{2}$ per horn. This gives

$$
M_{\text {array }} \propto n \frac{S^{2}}{\mathrm{NEP}_{\text {array }}^{2} / n},
$$

where $\mathrm{NEP}_{\text {array }}^{2}$ is the sum of $\mathrm{NEP}_{1}^{2}$ over all the horns, accounting for correlations in the noise. $\mathrm{NEP}_{\text {det }}$ is uncorrelated between detectors, so we can simply add the $\mathrm{NEP}_{\text {det }}^{2}$ contributions. For the photon noise, the Bose contribution is partly correlated between horns, but the Poisson contribution is always uncorrelated. To estimate the effect of noise correlations, I assume that the Bose noise is fully correlated over some patch in the focal plane and is completely uncorrelated outside that patch. Combining the various noise terms then gives

$$
\begin{aligned}
\mathrm{NEP}_{\mathrm{array}}^{2} \approx & n\left[\eta_{s}\left(\mathrm{NEP}_{\mathrm{cmb} P}^{2}+\mathrm{NEP}_{\mathrm{atm} P}^{2}\right)\right. \\
& \left.+\left(1-\eta_{s}\right) \mathrm{NEP}_{\mathrm{stop} P}^{2}+\mathrm{NEP}_{\mathrm{det}}^{2}\right] \\
& +\gamma_{\mathrm{cmb}}^{2} \Gamma_{\mathrm{cmb}} \eta_{s} \mathrm{NEP}_{\mathrm{cmb} B}^{2}+\gamma_{\mathrm{atm}}^{2} \Gamma_{\mathrm{atm}} \eta_{s} \mathrm{NEP}_{\mathrm{stop} B}^{2} \\
& +\gamma_{\mathrm{stop}}^{2} \Gamma_{\mathrm{stop}}\left(1-\eta_{s}\right) \mathrm{NEP}_{\mathrm{atm} B}^{2}
\end{aligned}
$$

where subscripts $P$ and $B$ indicate Poisson and Bose contributions. For the Bose terms, $\gamma$ is the number of horns in the patch of focal plane over which the Bose noise is fully correlated. $\Gamma$ is the number of independent samples in the FOV. If the radius of the correlated patch is $\rho$ and the radius of the telescope focal plane is $R$, then

$$
\begin{aligned}
\gamma & =\left(\frac{\rho}{a_{o}}\right)^{2} \text { for } a_{o} \leq \rho \\
& =1 \text { for } a_{o}>\rho,
\end{aligned}
$$

where $a_{o}$ is the outside diameter of a horn, and

$$
\begin{aligned}
\Gamma & =\left(\frac{R}{\rho}\right)^{2} \quad \text { for } a_{o} \leq \rho \\
& =\left(\frac{R}{a_{o}}\right)^{2} \quad \text { for } a_{o}>\rho .
\end{aligned}
$$

To check Eq. (8), consider first the case of horns that just fill the Airy disc and Bose noise that is correlated over the Airy disc, i.e., an array of essentially independent horns. There is one horn per Airy disc, so $\gamma \approx 1$ and $\Gamma \approx n$. The stop is fully, but not overly, illuminated; hence, $\eta_{s} \approx 1$. Equation ( $\underline{8}$ ) then gives $\mathrm{NEP}_{\text {array }}^{2} / n \approx$ $\mathrm{NEP}_{\text {cmb } P}^{2}+\mathrm{NEP}_{\text {atm_ } P}^{2}+\overline{N E P}_{\text {det }}^{2}+\mathrm{NEP}_{\text {cmb } B}^{2}+$ $\mathrm{NEP}_{\mathrm{atm}_{B}}^{2}$ which is, as expected, the same as $\mathrm{NEP}_{1}^{2}$ with

Table 1. Typical NEP Contributions in a Dual-Polarization, $\lambda=2 \mathrm{~mm}$ Receiver with $30 \mathrm{GHz}$ Bandwidth

\begin{tabular}{lcccrr}
\hline Noise Source & $T(\mathrm{~K})$ & $\epsilon$ & $\mathrm{NEP}_{P}\left(\mathrm{~W} \mathrm{~Hz}^{-1 / 2}\right)$ & $\mathrm{NEP}_{B}\left(\mathrm{WHz}^{-1 / 2}\right)$ \\
\hline CMB & 3 & 1 & $1.1 \times 10^{-17}$ & $3.5 \times 10^{-18}$ & 3.14 \\
Atmosphere + telescope & 300 & 0.03 & $3.9 \times 10^{-17}$ & $4.3 \times 10^{-17}$ \\
Cold stop & 8 & 1 & $2.9 \times 10^{-17}$ & $2.4 \times 10^{-17}$ \\
Detector & & & $10^{-17}$ & 1.21 \\
\hline
\end{tabular}


$\eta_{s}=1$ [see Eq. (2)]. This result also applies for horns that are much larger than the Airy disc $(\gamma=1, \Gamma=n$, and $\eta_{s}=1$ ). Now consider the case of horns that are much smaller than the Airy disc, again with Bose noise correlated over the Airy disc. If there are $m$ horns per Airy disc, $\gamma \approx m$, and the number of uncorrelated patches in the FOV is $\Gamma \approx n / m$. Each horn has $1 / m$ times the area of a horn that just fully illuminates the stop, so $\eta_{s} \approx 1 / m$. Since $m$ is large, $1-\eta_{s} \approx 1$. Equation ( ) then gives $\mathrm{NEP}_{\text {array }}^{2} / n \approx\left(\mathrm{NEP}_{\text {cmb_}}^{2} P^{+}\right.$ $\left.\mathrm{NEP}_{\text {atm } \_}^{2}\right) / m+\mathrm{NEP}_{\text {stop } P}^{2}+\mathrm{NEP}_{\text {det }}^{2}+\mathrm{NEP}_{\text {cmb } \_B}^{2}+$ $\mathrm{NEP}_{\text {atm } B}^{2}+m \mathrm{NEP}_{\text {stop_B }}^{2}$. The Poisson noise from the CMB and atmosphere is $m$ times smaller than for a single horn with $\eta_{s}=1$. This is because the power falling on each of the $m$ horns in the Airy disc is $m$ times smaller than the total power in the disc. The Poisson noise from the stop is not reduced by a factor of $m$ because the horns are small, so they all see the full noise contribution from the stop. The Bose noise from the CMB and atmosphere is correlated between horns in the Airy disc. The noise is, therefore, the same as for a single horn the size of the Airy disc. The stop Bose noise has an additional factor $m$, again because each horn sees the full noise from the stop.

For a distant source with angular diameter $\theta$, the Bose noise has a coherence factor $>1 / 2$ over a region of width $\sim \lambda / \theta[10]$. A patch of CMB that just fills the telescope beam gives a highly correlated region the size of the telescope. This region corresponds to an Airy disc in the focal plane. Bose noise from the CMB is therefore highly correlated over the Airy disc. A detailed calculation of the coherence factor for the stop noise depends on the geometry of the stop, but I can make a simple estimate that is useful for any stop configuration. Viewed from a horn, the stop subtends an angle $\sim 1 / F$, where $F$ is the focal ratio. Bose noise from the stop is, therefore, highly correlated over a region of width $\sim F \lambda$, i.e., about half the diameter of the Airy disc. The atmosphere is extended along the line of sight, and it is in the near field for a millimeterwave telescope larger than a few meters. The smeared atmosphere looks like a thermal source at the stop. Thus, the correlation properties of the Bose noise from the atmosphere are similar to those of the Bose noise from the stop. This picture is consistent with the performance of existing CMB instruments. These instruments have horns that are roughly the size of the Airy disc. They see the Bose noise as a degradation in the sensitivity of each receiver, but there is no evidence for a substantial degradation in array mapping speed [17]. Thus, the Bose noise cannot be highly correlated on scales much larger than the Airy disc. For the mapping speed estimates that follow, I will assume that the Bose noise from the CMB is fully correlated over the Airy disc and the Bose noise from the stop and atmosphere are fully correlated over a region half the diameter of the Airy disc, i.e., $\rho_{\mathrm{cmb}}=2 \rho_{\mathrm{atm}}=$ $2 \rho_{\text {stop }}=1.22 F \lambda$.

A typical millimeter-wave bolometer camera has detectors that are sensitive to both polarizations.
In this case, the mapping speed is given by Eq. (7) with $q=2$. A polarimeter has a separate detector for each polarization, and we take the difference between the two detector outputs. From Eqs. (3) and $(\underline{4}), \mathrm{NEP}_{B}^{2} \propto q^{2}$, so the Bose noise is fully correlated between polarizations (cf. $\mathrm{NEP}_{P}^{2} \propto q$ for the uncorrelated Poisson noise). Differencing rejects all the Bose noise contributions in Eq. (8), but $\mathrm{NEP}^{2}$ for the difference between the two detectors is twice that for a single detector. The noise for an array of polarimeters is therefore

$$
\begin{aligned}
\mathrm{NEP}_{\text {pol array }}^{2}(q=1)= & 2 n\left[\eta_{s}\left(\mathrm{NEP}_{\mathrm{cmb} \_P}^{2}+\mathrm{NEP}_{\mathrm{atm} \_}^{2}\right)\right. \\
& +\left(1-\eta_{s}\right) \mathrm{NEP}_{\text {stop } P}^{2} \\
& \left.+\mathrm{NEP}_{\text {det }}^{2}\right] .
\end{aligned}
$$

The mapping speed is given by Eq. (7) with $\operatorname{NEP}_{\text {pol array }}^{2}(q=1)$ for the noise. If differencing does not reject the Bose noise terms, the mapping speed for a polarimeter array is half that for a camera with $q=1$.

\section{Corrugated Horns}

In this section, I estimate the point-source mapping speed for an array of diffraction-limited corrugated horns. "Diffraction-limited" implies uniform phase across the horn aperture. This gives good coupling to a telescope focus, which also has a uniform phase across the Airy pattern. The aperture efficiency is

$$
\eta_{a}=\frac{\left|\int_{\text {horn }} E_{h} E_{a} \mathrm{~d} \mathbf{r}\right|^{2}}{\int_{\infty}\left|E_{h}\right|^{2} \mathrm{~d} \mathbf{r} \int_{\infty}\left|E_{a}\right|^{2} \mathrm{~d} \mathbf{r}}
$$

where

$$
\begin{aligned}
E_{h}(r) & =J_{0}\left(2.4048 \frac{r}{a_{h}}\right) \quad \text { for } r \leq a_{h} \\
& =0 \quad \text { for } r>a_{h}
\end{aligned}
$$

is the field at the horn aperture [18],

$$
E_{a}(r)=2 \frac{J_{1}(\pi r / F \lambda)}{\pi r / F \lambda}
$$

is the field in the Airy pattern [19], $a_{h}$ is the horn aperture radius, $\mathbf{r}$ is a radial vector in the field pattern, and $r=|\mathbf{r}|$. The optimum horn diameter is roughly equal to the diameter of the Airy disc. A smaller horn misses more of the power in the Airy pattern, so the aperture efficiency is lower. A larger horn underilluminates the stop, so the telescope beam gets larger and the aperture efficiency decreases.

The spillover efficiency is

$$
\eta_{s}=\frac{\int_{\text {stop }}\left|E_{s}\right|^{2} \mathrm{~d} \mathbf{r}}{\int_{\infty}\left|E_{s}\right|^{2} \mathrm{~d} \mathbf{r}},
$$

where $E_{s}$ is the field at the stop. To estimate $E_{s}$, I note that a corrugated horn has most of the power 
in the fundamental Gaussian mode, so $E_{s}(r) \propto$ $\exp -r^{2}$ [20]. The beam radius at the stop is $F \lambda a_{s} / a_{h}$, where $a_{s}$ is the stop radius. The stop is usually in the far field of the horn, so

$$
E_{s}(r) \approx \exp -\left(c \frac{r}{a_{s}} \frac{w \pi}{F \lambda}\right)^{2},
$$

where $w=0.6435 a_{h}$ is the beam waist diameter [18], and $c$ is a constant that gives the correct beam width at the stop. Choosing $c$ means making $\eta_{s}=\eta_{a}$ for small $a_{h}$. This leads to $c=0.67$.

Figure 1 shows $\eta_{a}$ and $\eta_{s}$ for a corrugated horn. A single receiver achieves peak mapping speed when the horn aperture efficiency is maximum. This corresponds to a corrugated horn aperture diameter of $2.4 F \lambda$, so the horn just fills the Airy disc. For a smooth-wall conical horn, the optimum aperture diameter is a little smaller at $2 F \lambda$ [13].

To calculate the mapping speed versus horn diameter for various receiver configurations, I substitute $\eta_{a}$ from Eq. (12) and $\eta_{s}$ from Eq. (15) into Eq. (7). The results are shown in Fig. 2 for $\lambda=2 \mathrm{~mm}$ corrugated horn arrays that fill the FOV at $F=4 / 3$. For these calculations, I used a FOV corresponding to $100 F \lambda$ in the focal plane. The results do not depend on the FOV as long as it is large compared with the horn aperture diameter. The noise contributions for Fig. 2 are given in Table 1 . The atmospheric transmission is for a good observing site, and the cold stop noise is appropriate for a receiver with a closed-cycle refrigerator system. Correlated Bose noise has a negligible effect on the mapping speed for this typical millimeter-wave receiver. The signal and correlated noise calculations account for the blockage due to the horn walls. For Fig. 2 , I used a wall thickness of $3 \lambda / 8$. The corrugations in the horn wall are $\lambda / 4$ deep, so the minimum metal thickness is $\lambda / 8$. At $\lambda=2 \mathrm{~mm}$, this is $0.25 \mathrm{~mm}$, which is reasonable for a small horn.

In Fig. 2, the camera mapping speed peaks at a horn aperture diameter of $1.6 F \lambda$. The mapping speed is degraded by $<10 \%$ for $1.3-2.0 F \lambda$ horns, i.e., a roughly $\pm 25 \%$ change in horn diameter.

Figure 3 shows the mapping speed for receivers with no detector noise and no stop noise. In this case,

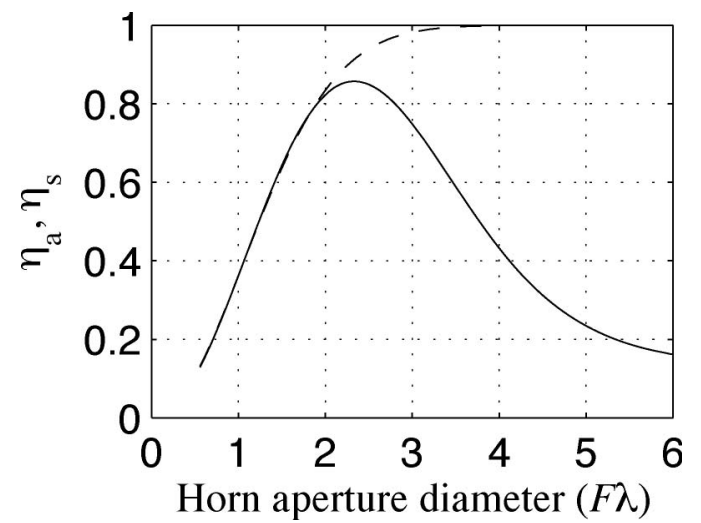

Fig. 1. Aperture efficiency $\eta_{a}$ (solid curve) and spillover efficiency $\eta_{s}$ (dashed curve) for a corrugated horn.

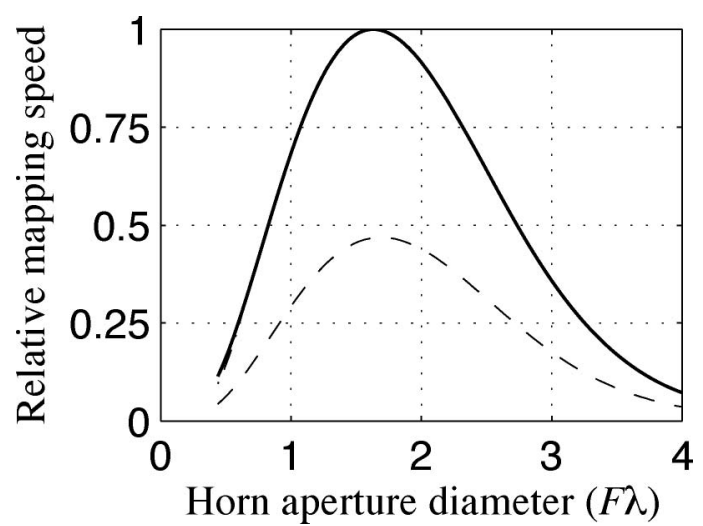

Fig. 2. Mapping speed for a camera with uncorrelated Bose noise (solid curve) from Eq. (7) with $q=2, \gamma=1$, and $\Gamma=n$. Mapping speed for a camera with Bose noise from the CMB correlated over an Airy disc and Bose noise from the stop and atmosphere correlated over a region half the diameter of the Airy disc (dashed-dotted curve, visible only at the bottom left) from Eq. (7) with $q=1$ and $\rho_{\text {cmb }}=2 \rho_{\text {atm }}=2 \rho_{\text {stop }}=1.22 F \lambda$. Also, mapping speed for a polarimeter array (dashed curve) from Eq. (7) with noise from Eq. (11). All the receiver arrays have corrugated horns filling the FOV of the telescope. Receiver and atmosphere parameters are given in Table 1 . The horn wall thickness is $3 \lambda / 8$ and $F=4 / 3$. Mapping speeds are relative to the peak for the camera with uncorrelated Bose noise. The signal for the polarimeter is half that for the camera.

the correlated noise from the atmosphere and CMB is a larger fraction of the total and it slightly degrades the mapping speed for small horns. The optimum horn diameter for a camera is $1.3 F \lambda$, which is smaller than in Fig. $\underline{2}$ because there is no detector and stop noise penalty for smaller horns.

Table 2 gives the optimum horn diameter for a range of horn wall thickness and beam focal ratio for a typical millimeter-wave receiver. Changes in wall thickness have only a small effect. The effect is bigger in faster systems because the horn wall is a larger fraction of the horn area. A smaller focal ratio gives a slightly larger optimum horn diameter, again because the wall is a larger fraction of the total area. For a reasonable wall thickness, e.g., $3 \lambda / 8$ to $\lambda / 2$ (cf. $\lambda / 4$ corrugation depth), the optimum horn diameter ranges from $1.6 F \lambda$ for a camera to $1.7 F \lambda$ for a polarimeter. If map points are combined to increase

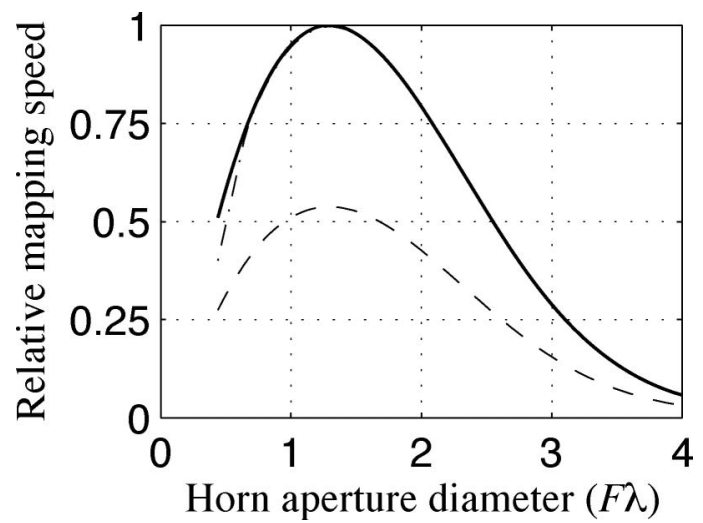

Fig. 3. Same as Fig. 2 but with $\mathrm{NEP}_{\text {stop }}=0$ and $\mathrm{NEP}_{\text {det }}=0$. The peak mapping speed here is $1.29 \times$ that in Fig. 2 . 
Table 2. Optimum Horn Diameter (in Units of $F \lambda$ ) Versus Wall Thickness and Focal Ratio ${ }^{a}$

\begin{tabular}{lccc}
\hline Wall Thickness & $F$ & Camera $^{b}$ & Polarimeter $^{c}$ \\
\hline 0 & - & 1.388 & 1.464 \\
$\lambda / 4$ & $4 / 3$ & 1.572 & 1.636 \\
$3 \lambda / 8$ & $4 / 3$ & 1.632 & 1.692 \\
$\lambda / 2$ & $4 / 3$ & 1.680 & 1.736 \\
$\lambda / 4$ & 2 & 1.524 & 1.592 \\
$3 \lambda / 8$ & 2 & 1.572 & 1.636 \\
$\lambda / 2$ & 2 & 1.616 & 1.676 \\
\hline
\end{tabular}

${ }^{a}$ Receiver and atmosphere parameters from Table 1.

${ }^{b}$ Equation (7) with $q=2$ and $\rho_{\mathrm{cmb}}=2 \rho_{\text {atm }}=2 \rho_{\text {stop }}=1.22 F \lambda$.

${ }^{c}$ Equation (ㄱ) with noise from Eq. (11), i.e., no Bose noise contributions.

the signal-to-noise ratio on the point source, the optimum horn diameter will be a little smaller. The optimum diameter for smooth-wall conical horns is smaller than for corrugated horns because smoothwall horns have a wider field pattern and do not require a thick wall for corrugations.

\section{Conclusions}

For an array of large horns, the point-source mapping speed drops rapidly with increasing horn diameter because the telescope beam gets bigger and the number of detectors in the FOV gets smaller. Small horns suffer a sensitivity penalty because more of the beam falls on the cold stop and each detector and its associated readout contributes noise. At millimeter wavelengths, correlated noise slightly degrades the mapping speed for small horns if the detector noise and stop noise are both small. At submillimeter wavelengths, Poisson noise dominates for both the stop and CMB. The effect of correlated noise is negligible at these shorter wavelengths.

For an array of corrugated horns, and typical millimeter-wave receiver parameters, the optimum horn diameter is $1.6-1.7 F \lambda$. For ideal receivers, with no detector noise and no stop noise, the optimum horn diameter is roughly $1.3 F \lambda$. The mapping speed is degraded by $<10 \%$ for a $\pm 25 \%$ change in horn diameter about the optimum.

This work was supported by the National Science Foundation (NSF) under grant ANT-0638937, and by the John B. and Nelly Kilroy Foundation. The author thanks T. Crawford and J. McMahon for helpful discussions. The author also thanks the referees for their comments, which led to significant improvements in the paper.

\section{References}

1. P. J. B. Clarricoats and A. D. Olver, Corrugated Horns for Microwave Antennas (Peter Peregrinus, 1984).

2. W. Hu and S. Dodelson, "Cosmic microwave background anisotropies," Annu. Rev. Astron. Astrophys. 40, 171-216 (2002).

3. M. Zaldarriaga, "The polarization of the cosmic microwave background," in Measuring and Modeling the Universe, Carnegie Observatories Astrophysics Series, W. L. Freedman, ed. (Cambridge U. Press, 2003), Vol. 2.
4. U. Seljak and M. Zaldarriaga, "Signature of gravity waves in the polarization of the microwave background," Phys. Rev. Lett. 78, 2054-2057 (1997).

5. M. Zaldarriaga and U. Seljak, "Gravitational lensing effect on cosmic microwave background polarization,” Phys. Rev. D 58, 023003 (1998).

6. K. W. Yoon, P. A. R. Ade, D. Barkats, J. O. Battle, E. M. Bierman, J. J. Bock, J. A. Brevik, H. C. Chiang, A. Crites, C. D. Dowell, L. Duband, G. S. Griffin, E. F. Hivon, W. L. Holzapfel, V. V. Hristov, B. G. Keating, J. M. Kovac, C. L. Kuo, A. E. Lange, E. M. Leitch, P. V. Mason, H. T. Nguyen, N. Ponthieu, Y. D. Takahashi, T. Renbarger, L. C. Weintraub, and D. Woolsey, "The Robinson Gravitational Wave Background Telescope (BICEP): a bolometric large angular scale CMB polarimeter," Proc. SPIE 6275, 62751K (2006).

7. J. R. Hinderks, P. Ade, J. Bock, M. Bowden, M. L. Brown, G. Cahill, J. E. Carlstrom, P. G. Castro, S. Church, T. Culverhouse, R. Friedman, K. Ganga, W. K. Gear, S. Gupta, J. Harris, V. Haynes, B. G. Keating, J. Kovac, E. Kirby, A. E. Lange, E. Leitch, O. E. Mallie, S. Melhuish, Y. Memari, J. A. Murphy, A. Orlando, R. Schwarz, C. O'Sullivan, L. Piccirillo, C. Pryke, N. Rajguru, B. Rusholme, A. N. Taylor, K. L. Thompson, C. Tucker, A. H. Turner, E. Y. S. Wu, and M. Zemcov, "QUaD: a high-resolution cosmic microwave background polarimeter," Astrophys. J. 692, 1221-1246 (2009).

8. P. Oxley, P. Ade, C. Baccigalupi, P. deBernardis, H.-M. Cho, M. J. Devlin, S. Hanany, B. R. Johnson, T. Jones, A. T. Lee, T. Matsumura, A. D. Miller, M. Milligan, T. Renbarger, H. G. Spieler, R. Stompor, G. S. Tucker, and M. Zaldarriaga, "The EBEX experiment," Proc. SPIE 5543, 320-331 (2004).

9. R. H. Brown and R. Q. Twiss, "Interferometry of the intensity fluctuations in light. I. basic theory: the correlation between photons in coherent beams of radiation," Proc. R. Soc. A 242, 300-324 (1957).

10. R. H. Brown and R. Q. Twiss, "Interferometry of the intensity fluctuations in light. II. an experimental test of the theory for partially coherent light," Proc. R. Soc. A 243, 291-319 (1958).

11. J. M. Lamarre, "Photon noise in photometric instruments at far-infrared and submillimeter wavelengths," Appl. Opt. 25, 870-876 (1986)

12. J. Zmuidzinas, "Thermal noise and correlations in photon detection," Appl. Opt. 42, 4989-5008 (2003).

13. M. W. Griffin, J. J. Bock, and W. K. Gear, "Relative performance of filled and feedhorn-coupled focal-plane architectures," Appl. Opt. 41, 6543-6554 (2002).

14. D. J. Benford, T. R. Hunter, and T. G. Phillips, "Noise equivalent power of background limited thermal detectors at submillimeter wavelengths," Int. J. Infrared Milli. Waves 19, 931-938 (1998).

15. P. L. Richards, "Bolometers for infrared and millimeter waves," J. Appl. Phys. 76, 1-24 (1994).

16. P. Fellgett, R. Clark Jones, and R. Q. Twiss, "Fluctuations in photon streams," Nature 184, 967-969 (1959).

17. M. C. Runyan, P. A. R. Ade, R. S. Bhatia, J. J. Bock, M. D. Daub, J. H. Goldstein, C. V. Haynes, W. L. Holzapfel, C. L. Kuo, A. E. Lange, J. Leong, M. Lueker, M. Newcomb, J. B. Peterson, C. Reichardt, J. Ruhl, G. Sirbi, E. Torbet, C. Tucker, A. D. Turner, and D. Woolsey, "ACBAR: the Arcminute Cosmology Bolometer Array Receiver,” Astrophys. J. Suppl. Ser. 149, 265-287 (2003).

18. P. F. Goldsmith, Quasioptical Systems (IEEE, 1998), Chaps. 6 and 7.

19. M. Born and E. Wolf, Principles of Optics, 7th ed. (Cambridge U. Press, 1999), p. 439.

20. R. J. Wylde, "Millimetre-wave Gaussian beam-mode optics and corrugated feed horns," Proc. IEEE Part H Microwaves Antennas Propag. 131, 258-262 (1984). 